\title{
Accurate Prediction Method of Breakdown Voltage in Air at Atmospheric Pressure
}

\author{
Nam-Kyung Kim*, Se-Hee Lee*, G. E. Georghiou**, \\ Dong-Wook Kim* and Dong-Hun Kim ${ }^{\dagger}$
}

\begin{abstract}
To predict accurately the breakdown voltage in air at atmospheric pressure, a fully coupled finite element analysis combining the hydrodynamic diffusion-drift equations with Poisson's equation is proposed in the current paper. As three kinds of charged transport particles are nonlinearly coupled with spatial electric fields, the equations should be solved by an iterative numerical scheme, in which secondary effects, such as photoemission and photoionization, are considered. The proposed method has been successfully applied to evaluate the breakdown voltage in circular parallel-plane electrodes. Its validity has been proved through the comparison of the predicted and experimental results. The effects of numerical conditions of the initial charge, photoemission, and background ionization on the discharge phenomena are quantitatively assessed through Taguchi's design of experiment method.
\end{abstract}

Keywords: Electric breakdown, Electron emission, Finite element method, Gas discharges

\section{Introduction}

In the development of electric systems, such as highvoltage apparatuses and micro-electromechanical devices, the breakdown voltage is likely to become a critical issue in achieving robustness of the system designed. Breakdown voltage is usually estimated from empirical data or maximum electric field values calculated by electromagnetic analysis tools. These approaches can only give engineers a global trend of the breakdown voltage in a system. However, to obtain in-depth knowledge of the breakdown, designers need to determine the local characteristics, such as discharge evolution, discharge path, continuous discharge pattern, densities of ions and electrons, etc.

In a quest to explore discharge phenomena, the finite difference method or finite element method (FEM), coupled with the method of characteristics or flux corrected transport method, has been used to date [1-8]. The governing equations for the discharge analysis are generally composed of two kinds of differential equations: the first-order charge transport equation and the secondorder electric field equation. The convergence of numerical solutions is strongly dependent on the convective term in the charge transport equation. In such convectivedominated transport problems, instabilities may occur in numerical solutions. These instabilities may be noted as

$\dagger \quad$ Corresponding Author: Dept. of Electrical Engineering, Kyungpook National University, Korea. (dh29kim@ee.knu.ac.kr)

* Dept. of Electrical Engineering, Kyungpook National University, Korea. (sanum@nate.com,shlees@knu.ac.kr,bloodkdw@nate.com)

** Dept. of Electrical and Computer Engineering, University of Cyprus, Nicosia 1678, Cyprus.

Received: August 10, 2010; Accepted: September 23, 2011 oscillations and exhibit divergence in field solutions. Instabilities can be easily observed in front of the moving charges where steep field gradients appear. To avoid these problems, in the current study, the cell Peclet number is carefully controlled by generating fine meshes around the discharge column between electrodes.

To simulate the breakdown situation more accurately, secondary effects, such as the photoemission, background ionization, and photoionization, should be considered during the discharge process. In the current paper, the mechanism of photoemission and background ionization is embodied in the boundary conditions. The fully coupled finite element model is successfully applied to the prediction of the breakdown voltage and to the analysis of discharge phenomena in circular parallel-plane electrodes. Whereas the effects of the initial charge distribution, photoemission, and background ionization on the discharge phenomena were qualitatively discussed in [1], they are quantitatively assessed in this paper through Taguchi's design of experiment (DOE) method.

The proposed numerical setup is implemented using the COMSOL Multiphysics software and MATLAB programming environment, with the two packages constantly communicating with each other and exchanging information required for discharge simulation.

\section{Fully Coupled Hydrodynamic Diffusion-Drift Models for Discharge Analysis in Air}

To analyze the discharge phenomena in air, the fully coupled hydrodynamic diffusion-drift models are 
employed with the unknown variables of electron density $\left(N_{e}\right)$, positive ion density $\left(N_{p}\right)$, negative ion density $\left(N_{n}\right)$, and electric scalar potential $(V)[4]$.

$$
\begin{aligned}
\frac{\partial N_{e}}{\partial t}=S+N_{e} \alpha\left|\mathbf{W}_{\mathbf{e}}\right|-N_{e} \eta\left|\mathbf{W}_{\mathbf{e}}\right|-N_{e} N_{p} \beta_{e p}-\nabla \cdot\left(N_{e} \mathbf{W}_{\mathbf{e}}\right) \\
+\nabla \cdot\left(D \nabla N_{e}\right) \\
\frac{\partial N_{p}}{\partial t}=S+N_{e} \alpha\left|\mathbf{W}_{\mathbf{e}}\right|-N_{e} N_{p} \beta_{e p}-N_{n} N_{p} \beta_{n p}-\nabla \cdot\left(N_{p} \mathbf{W}_{p}\right) \\
\frac{\partial N_{n}}{\partial t}=N_{e} \eta\left|\mathbf{W}_{\mathbf{e}}\right|-N_{n} N_{p} \beta_{n p}-\nabla \cdot\left(N_{n} \mathbf{W}_{n}\right) \\
\nabla \cdot(-\varepsilon \nabla V)=e\left(N_{p}-N_{e}-N_{n}\right)
\end{aligned}
$$

where $t$ is the time, $\mathbf{W}_{\mathrm{e}}, \mathbf{W}_{\mathrm{p}}$, and $\mathbf{W}_{\mathrm{n}}$ are the electron, positive ion, and negative ion drift velocities, respectively, and $D$ is the electron diffusion coefficient. The symbols $\alpha$, $\eta$, and $\beta$ denote the ionization, attachment, and recombination coefficients, respectively. The term $S$ represents the source term due to photoionization. In the discharge simulation, the material functions are used similarly to those presented in [5]. The discharge current is calculated by the generalized energy approach, which yields reliable results when combined with the FEM [6-8].

The transport equations strongly depend on the convective term. The discretization of convectivedominated transport problems can lead to instabilities in numerical solutions. In case a relatively short gap between electrodes is considered, the dominant carrier with high speed is the electron; the ions then move very slowly. Thus, the electrons mainly cause the numerical instability. The instabilities may be noted as the oscillation or divergence of the field solutions in front of the moving electrons where a steep field gradient is observed. To check the instability, the Peclet number $P_{e}$ is introduced [9].

$$
P_{e}=h|\boldsymbol{\beta}| / c
$$

where $h$ is the local mesh diameter, $\boldsymbol{\beta}$ is the convective velocity vector corresponding to $\mathbf{W}_{\mathrm{e}}$, and $c$ denotes the diffusivity. The numerical solution becomes unstable when the Peclet number exceeds two [9]. In our simulation, the elements are generated with the criteria of $P_{e}<0.4$.

\section{Secondary Effects of Streamer Propagation}

In the current paper, the secondary effects in insulator materials, such as photoemission and photoionization, on gas discharge, specifically on the streamer development and propagation, are executed based on the twodimensional axisymmetric FEM algorithm.

To evaluate the amount of electrons emitted from the cathode because of the photoemission mechanism, the photonic sources are integrated over the whole discharge space divided into additional rectangular grids, as shown in Fig. 1. The photoemission effect is given by the following equation from [10]:

$$
N_{e}^{s}=\frac{\gamma_{p} N_{e 1} \alpha_{1}^{\prime}\left|\mathbf{W}_{\mathbf{e}}\right| V_{1}}{\left|\mathbf{W}_{\mathbf{e s}}\right| 8 \pi^{2}} \int_{0}^{2 \pi} \frac{z}{c^{3}} e^{-\mu c} d \theta
$$

where $N_{e}^{s}$ is the electron density emitted from the segment $d A, \gamma_{p}$ is the photoemission coefficient, $N_{\mathrm{e} 1}$ is the electron density emitted from the volume element $V_{1}, \alpha_{1}^{\prime}$ is the photoemission coefficient, $\mathbf{W}_{\mathrm{es}}$ is the electron drift speed on the cathode surface, $\mu$ is the absorption coefficient, and $z$ and $c$ are the geometric displacements, respectively, as illustrated in Fig. 1.

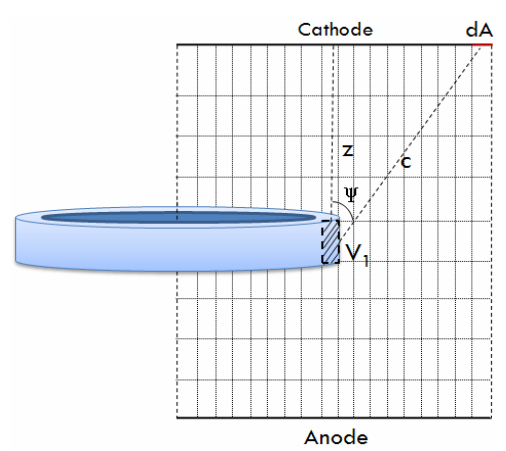

Fig. 1. Schematic representation for evaluating photoemission from the cathode caused by volume $\mathrm{V}_{1}$. The grids in dotted lines represent the small volumes of photonic sources.

To evaluate directly the magnitude of photoionization only at a certain position of the discharge space, the integration over the whole space of interest should be carried out by the photoionization function presented in [11]. Such process requires much computing time as well as different secondary grids in three dimensions. Thus, the background ionization scheme is adopted in the current study to pursue computational efficiency without loss of simulation accuracy. A background of charged particles is provided in the gap as an effective secondary mechanism for the propagation of the cathode-directed streamer. This mechanism is implemented in the model by a constant value source term.

\section{Numerical Results}

\subsection{Verification of numerical setup}

To verify our numerical setup, the circular parallel-plane electrodes in Fig. 2(a) are tested. The fine meshes are 
distributed along the $\mathrm{z}$-axis, and the cell Peclet number is set to less than 0.4, as shown in Fig. 2(b). The pressure of air is 760 Torr, and the temperature is $300 \mathrm{~K}$. The number of mesh elements and nodes used is 9804 and 5144, respectively. The initial charge distribution is located at $0.003 \mathrm{~cm}$ from the cathode and is in the form of a Gaussian distribution:

$$
\left.N_{e}\right|_{t=0}=\left.N_{p}\right|_{t=0}=N_{0} \exp \left[-\left(\frac{r-r_{0}}{0.006}\right)^{2}-\left(\frac{z-z_{0}}{0.006}\right)^{2}\right]
$$

where $N_{0}$ is the initial charge density of $10 \mathrm{~cm}^{-3}$, (i.e., number of electrons and positive ions), and $r_{0}$ and $z_{0}$ are the center coordinates of the initial charge distribution.
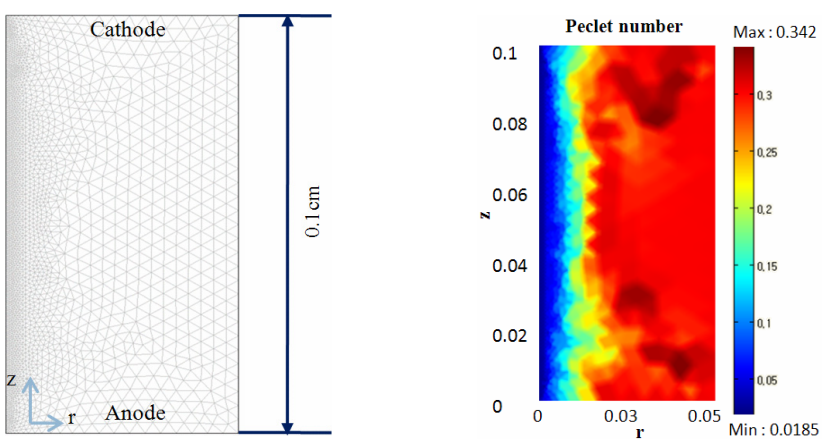

(a) Mesh distribution

(b) Magnitude of the Peclet number

Fig. 2. Analysis model with mesh distribution and the Peclet number

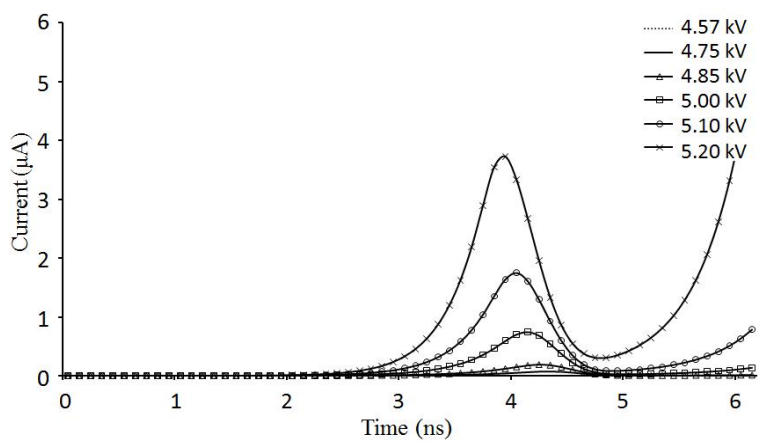

Fig. 3. Current profiles for different voltages applied to electrodes

Fig. 3 depicts the current during the evolution from the avalanche to the streamer propagation when the applied voltage increases. The breakdown onset voltage is predicted around $4.85 \mathrm{kV}$ by the proposed method. In all cases of the onset voltage, a streamer develops and propagates, but note that there is a considerable time delay depending on the applied voltage [12]. This delay stems from the fact that when the first avalanche is absorbed at the anode, there is a time delay for the second avalanche to move from the cathode region, as it depends on the background field intensity. Although the predicted result has an error of $6 \%$ compared with the experimental onset voltage of $4.56 \mathrm{kV} \mathrm{[1],} \mathrm{the} \mathrm{terminal} \mathrm{current} \mathrm{profile}$ predicted is similar to the experimental one presented in [13].

\subsection{Secondary effects on streamer propagation}

To investigate the secondary effects on the streamer propagation characteristics, three possible combinations from the two secondary processes (i.e., photoemission and background ionization) are tested. The magnitude of background ionization is set to $10^{7} \mathrm{~cm}^{-3}$; the other simulation conditions remain the same. Fig. 4 shows the current profiles when the different secondary effects are applied respectively. When both effects are included, the fastest streamer propagation is observed among them. After several nanoseconds, the streamer is fully developed between the electrodes, and the breakdown phenomena appear in all cases. With the propagation of the cathodedirected streamer, the variations of the electron density and the spatial electric field distribution are presented in Figs. 5 and 6 , respectively.

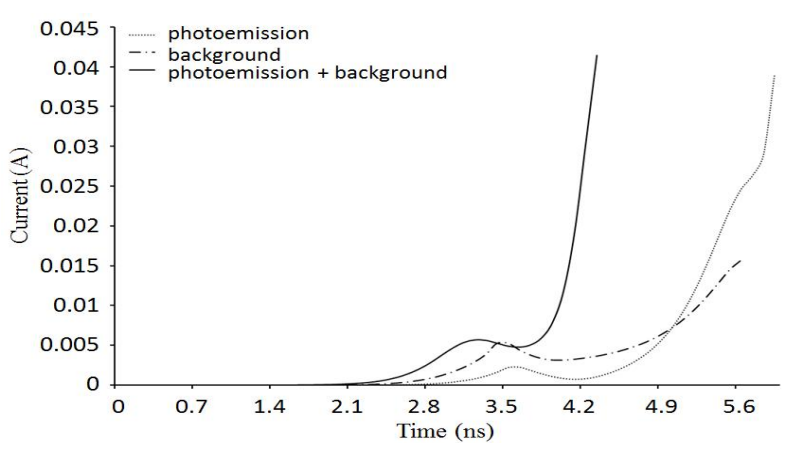

Fig. 4. Current profiles with the different secondary effects

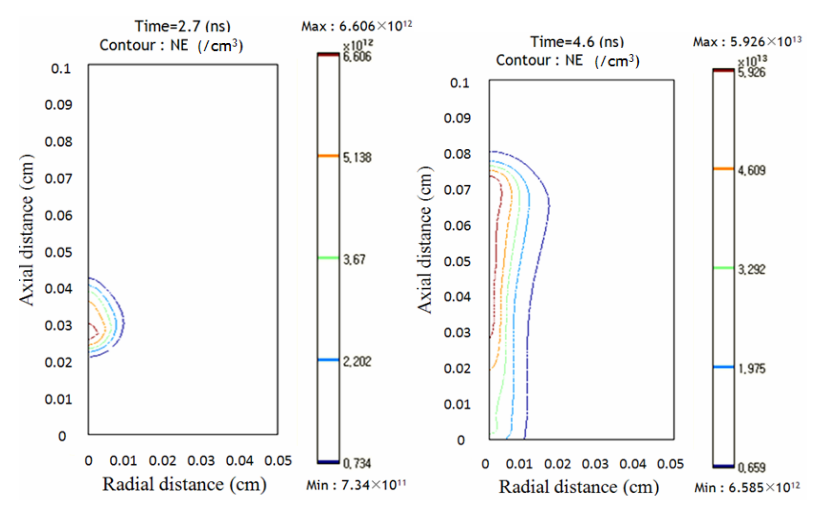

Fig. 5. Electron density at 2.7 (ns) and 4.6 (ns) including all secondary effects where the discharge channel is formed around the $\mathrm{z}$-axis 


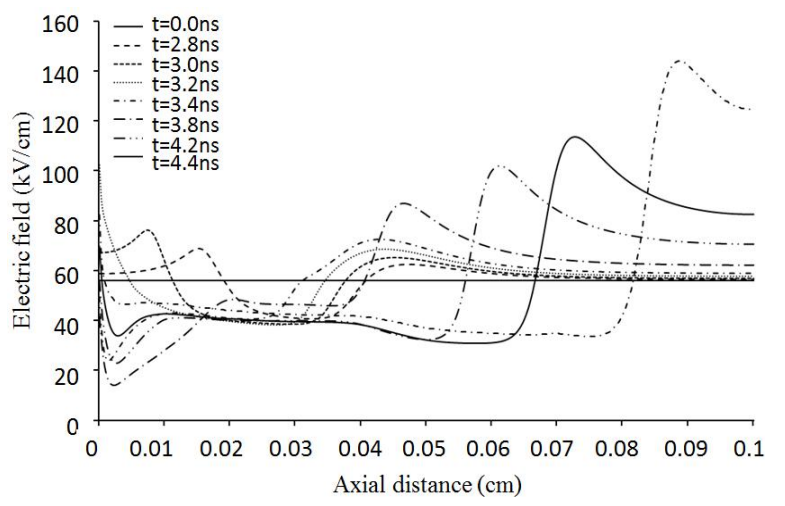

Fig. 6. Electric field distribution along the z-axis from the anode to cathode

\subsection{Assessment of secondary effects for simulation parameters}

To assess quantitatively the effect of each of the various simulation conditions (e.g., photoemission coefficient, background ionization value, and initial charges) on the streamer propagation characteristic, Taguchi's DOE method is introduced [14-16].

The photoemission coefficient, background ionization, and number of initial charges (i.e., electron-positive ion pairs) are set as the design parameters, as shown in Table 1. Based on the number of design parameters and the settings considered, a standard orthogonal array L9 is selected for the matrix numerical experiment in Table 2. In this array, the columns are assumed mutually orthogonal, and the three factors, A, B, and C, correspond to the three design parameters with three levels [i.e., photoemission coefficient (A), background ionization (B), and number of initial charges (C) used on discharge simulation]. The performance, $y_{i}$, in (8), among the nine combinations of design parameters, is evaluated in terms of the delay time required for reaching a certain current level of $0.1 \mathrm{~mA}$ in Table 2. The sensitivity of each design parameter and its performance are represented by the value of SN ratio. For "the smaller the better" case, which resembles the minimization of the performance (i.e., delay time), the SN ratio can be calculated by

$$
\text { SN ratio }=-10 \log \left(\sum y_{i}^{2} / n\right)
$$

where $n$ denotes the experiment number belonging to the $i$ th row in Table 2 .

Based on the results in Table 2, Analysis of Means (ANOM) and ANOVA [11-13] are performed to estimate the effects of the three design parameters and to determine the relative importance of each one. Fig. 7 shows the overall mean of the $\mathrm{SN}$ ratios of all design factor levels for the delay time. To reduce the delay time, ANOM suggests the C3 level (the number of initial charges) is the best choice of control factor, denoted as $\mathrm{C}$ here, as it corresponds to the highest average $\mathrm{SN}$ ratio. In other words, the larger the initial charges are, the larger the contribution to shortening the delay time to the other factors considered.

Table 1. L9 $\left(3^{4}\right)$ Orthogonal array and design parameters for the first setup

\begin{tabular}{c|c|c|c}
\hline Experiments & $\mathrm{A}$ & $\mathrm{B}$ & $\mathrm{C}$ \\
\hline 1 & 0.001 & $10^{6}$ & 10 \\
\hline 2 & 0.005 & $10^{7}$ & 100 \\
\hline 3 & 0.010 & $10^{8}$ & 1000 \\
\hline
\end{tabular}

where A denotes the photoemission coefficient, B the background ionization, and $\mathrm{C}$ the number of initial charges.

Table 2. SN ratios calculated by nine experiments through the evaluation function (time) and MSD

\begin{tabular}{c|c|c|c|c|c|c}
\hline Experiments & $\mathrm{A}$ & $\mathrm{B}$ & $\mathrm{C}$ & $\begin{array}{c}\text { Time }( \\
\mathrm{ns})\end{array}$ & $\begin{array}{c}\text { MSD } \\
\left(\mathrm{X} 10^{-17}\right)\end{array}$ & SN Ratio \\
\hline 1 & 1 & 1 & 1 & 5.15 & 2.65 & 165.76 \\
\hline 2 & 1 & 2 & 2 & 4.35 & 1.89 & 167.23 \\
\hline 3 & 1 & 3 & 3 & 3.63 & 1.32 & 168.80 \\
\hline 4 & 2 & 1 & 3 & 3.56 & 1.27 & 168.97 \\
\hline 5 & 2 & 2 & 1 & 4.61 & 2.13 & 166.73 \\
\hline 6 & 2 & 3 & 2 & 3.86 & 1.49 & 168.27 \\
\hline 7 & 3 & 1 & 2 & 4.01 & 1.61 & 167.94 \\
\hline 8 & 3 & 2 & 3 & 3.44 & 1.18 & 169.27 \\
\hline 9 & 3 & 3 & 1 & 4.02 & 1.62 & 167.92 \\
\hline
\end{tabular}

where Time denotes the specific time to reach $0.1 \mathrm{~mA}$, MSD denotes the mean square deviation, and $\mathrm{SN}$ ratio denotes the signal-to-noise ratio that can be calculated by ( 8$)$.

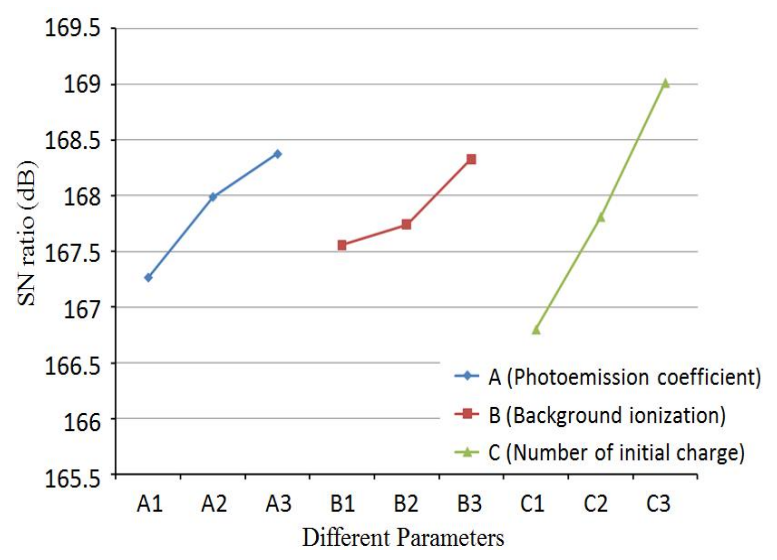

Fig. 7. Sensitivity of each parameter factor

Fig. 8 presents the relative importance of various design parameters with respect to the delay time through ANOVA, which is strongly analogous to Fourier analysis that provides the amplitude and power of a harmonic. As seen in the result, evidently, the order of importance of the considered design parameters is $\mathrm{C}, \mathrm{A}$, and $\mathrm{B}$. Based on these results, the large number of initial electron-positive ion pairs and appropriate photoemission coefficient are of paramount importance in shortening the delay time to reach the second avalanche. 


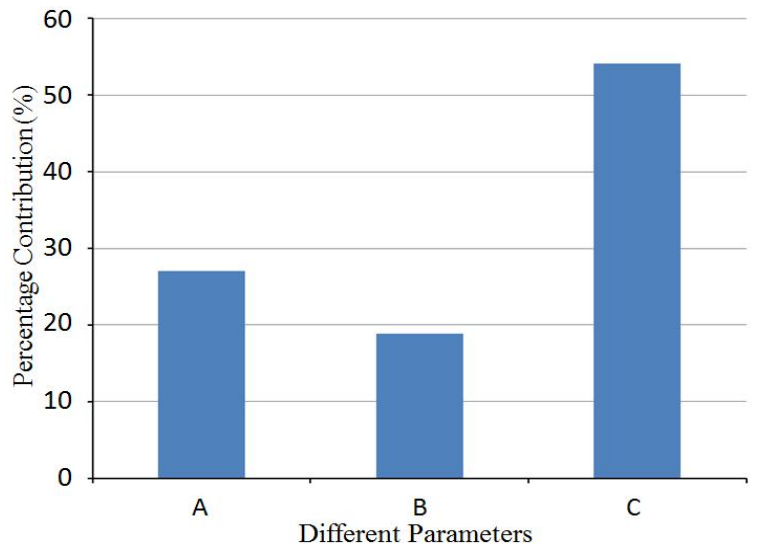

Fig. 8. Effects of each parameter on the streamer propagation characteristic

\section{Conclusion}

The fully coupled FEM combining the particle transport equations with the Poisson's equation has been presented for accurately predicting the breakdown voltage in air at atmospheric pressure. To avoid numerical instability, the meshes are carefully generated around the streamer channel based on the cell Peclet number. The validity of the proposed method has been proved through the comparison of the predicted and experimental breakdown onset voltages. Moreover, the effects of numerical conditions of the initial charge, photoemission, and background ionization on discharge phenomena are quantitatively assessed through Taguchi's DOE method.

\section{Acknowledgments}

This research was supported by Basic Science Research Program through the National Research Foundation of Korea (NRF) funded by the Ministry of Education, Science, and Technology (2011-0029721).

\section{References}

[1] G. E. Georghiou, R. Morrow and A. C. Metaxas, "Two-dimensional simulation of streamers using the FE-FCT algorithm," J. Phys. D: Appl. Phys. vol. 33, pp. L27-L32, 2000.

[2] J. P. Boris, "Flux-corrected transport I. SHASTA, A fluid transport algorithm that works," J. Comput. Phys., vol. 11, pp. 38-69, 1973.

[3] S. T. Zalezak, "Fully multidimensional flux-corrected transport algorithms for fluids," J. Comput. Phys., vol. 31, pp. 335-362, 1979.

[4] R. Morrow and J. J. Lowke, "Streamer propagation in air," J. Phys. D: Appl. Phys. vol. 30, pp. 614-627,
1997.

[5] G. E. Georghiou, et al., "Numerical Modeling of atmospheric pressure gas discharges leading to plasma production," J. Phys. D: Appl. Phys. vol. 38, pp. R303-R328, 2005.

[6] W. Min, et al., "An investigate of FEM-FCT method for streamer corona simulation," IEEE Trans Magn., vol. 36, pp. 1280-1284, 2000.

[7] B. Khaddour, et al., "Numerical solution and experiment test for corona discharge between blade and plate," IEEE Trans Magn., vol. 43, pp. 11931196, 2007.

[8] Se-Hee Lee, Se-Yeon Lee, Young-Ki Chung and IlHan Park, "Finite-Element Analysis of Corona Discharge Onset in Air With Artificial Diffusion Scheme and Under Fowler-Nordheim Electron Emission," IEEE Trans Magn., vol. 43, pp. 14531456, 2007.

[9] O. C. Zienkiewicz, R. L. Taylor, and P. Nithiarasu, The Finite Element Method for Fluid Dynamics, 6th edition, Elsevier, 2005.

[10] G. E. Georghiou, R. Morrow and A. C. Metaxas, "The effect of photoemission on the streamer development and propagation in short uniform gaps," J. Phys. D: Appl. Phys. vol. 34, pp. 200-208, 2001.

[11] A. Hallac, G. E. Georghiou and A. C. Metaxas, "Secondary emission effects on streamer branching in transient non-uniform short-gap discharge," J. Phys. D: Appl. Phys. vol. 36, pp. 2498-2509, 2003.

[12] G. E. Georghiou, R. Morrow and A. C. Metaxas, "Characterization of point-plane corona in air at radio frequency using the FE-FCT method," J. Phys D: Appl. Phys., vol. 32, pp. 2204-2218, 1999.

[13] J.M. Meek and J. D. Craggs, Electrical Breakdown of Gasses, John Wiley \& Sons, Ltd., 1978.

[14] S. X. Chen, T. S. Low, and B. Bruhl, "The robust design approach for reducing cogging torque in permanent magnetic motors", IEEE Trans Magn., vol. 34, pp. 2135-2137, 1998.

[15] H. T. Wang, Z. J. Liu, S. X. Chen, and J. P. Yang, "Application of Taguchi method to robust design of BLDC motor performance", IEEE Trans Magn., vol. 35, pp. 3700-3702, 1999.

[16] T. Low, S. Chen, and X. Gao, "Robust torque optimization for BLDC spindle motors", IEEE Trans Magn., vol. 48, pp. 656-663, 2001.

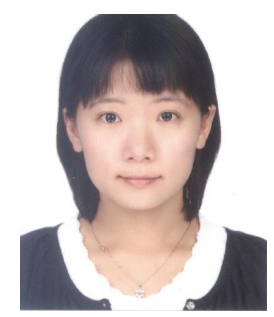

Nam-Kyung Kim was born in Korea in 1983. She received her B.S. and M.S. degrees from the Department of Electrical Engineering of Kyungpook National University, Daegu, Korea, in 2007 and 2010, respectively. She is currently taking her Ph.D. at Kyungpook National University. 


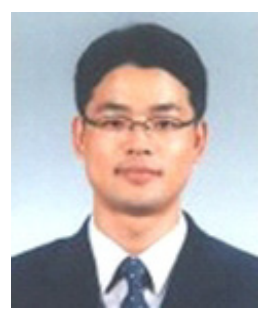

See-He Lee received his B.S. and M.S. degrees in Electrical Engineering from Soongsil University, Seoul, Republic of Korea, in 1996 and 1998, respectively. He received his Ph.D. degree in Electrical and Computer Engineering from Sungkyunkwan University in 2002. He conducted his postdoctoral research at the Massachusetts Institute of Technology (MIT) and worked for the Korea Electrotechnology Research Institute (KERI) before joining the faculty of Kyungpook National University in the School of Electrical Eng. and Computer Science in 2008. His research interests include analysis and design of electromagnetic multiphysics problems spanning the macro- to the nano-scales.

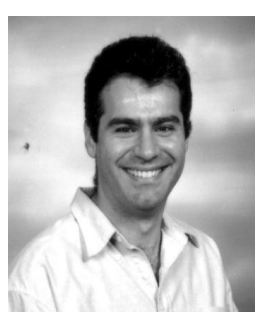

George Georghiou graduated from the University of Cambridge with a BA (1995), MEng (1996), and MA (1997), all with distinction, and $\mathrm{PhD}$ (1999). He continued his work at the University of Cambridge as a Research Fellow at Emmanuel College for three years (1999-2002). He is currently the undergraduate course leader in Electrical Engineering at the University of Southampton, Department of Electronics and Computer Science, and a Research Advisor for the Electricity Utilization, University of Cambridge. His research interests lie predominantly in the utilization of electromagnetic fields and plasma processes for environmental, food processing and biomedical applications, BioMEMS, Nanotechnology, and Power Systems.

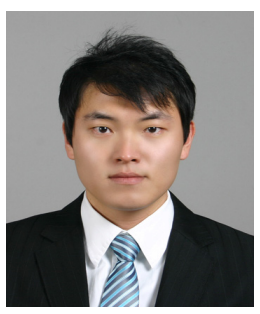

Dong-Wook Kim was born in Korea in 1982. He received his B.S. degree from the Department of Electrical Engineering of Kyungpook National University, Daegu, Korea, in 2009. He is currently taking his master's course at Kyungpook National University.

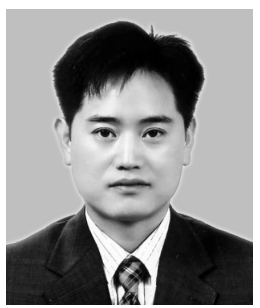

Dong-Hun Kim received his M.Sc. and Ph.D. degrees in Electrical Engineering from Seoul National University, Seoul, Republic of Korea, in 1994 and 1998, respectively. He was a Senior Researcher at the Digital Appliance Research Center of LG Inc. in Seoul, Republic of Korea, from 1998 to 2001. He continued his research at the University of Southampton in the United Kingdom as a Research Fellow for two years (2002-2003). He is currently an Associate Professor at the Department of Electrical Engineering at Kyungpook National University, Daegu, Republic of Korea. His main interests include electromagnetic field analysis and design optimization of electrical appliances and biomedical application. 